\title{
Educational challenges faced by international medical graduates in the UK
}

This article was published in the following Dove Press journal:

Advances in Medical Education and Practice

29 June 2017

Number of times this article has been viewed

\author{
Ahmed Hashim \\ Gastroenterology Department, \\ Brighton and Sussex University \\ Hospitals NHS Trust, Brighton, UK
}

Correspondence: Ahmed Hashim Gastroenterology Department, Brighton and Sussex University Hospitals NHS Trust, Eastern Road, Brighton BN2 5BE, UK

Email ahmedsir37@hotmail.com
Introduction: International medical graduates (IMGs) in the UK constitute approximately one-quarter of the total number of doctors registered in the General Medical Council (GMC). The transition of IMGs into the health care system in the UK is accompanied by significant sociocultural and educational challenges. This study aims to explore the views of IMGs in medical training on the educational challenges they face.

Methods: This study was conducted in the Kent, Surrey and Sussex region in 2015. All IMGs who work in medical (physicianly) training programs were included. Data were collected through a questionnaire and semi-structured interviews. Thematic approach was used to analyze the qualitative data. Results: Of the total 61 IMGs included, 17 responded to the survey and 3 were interviewed. The common educational barriers faced by IMGs were related to lack of appreciation of the values and structure of the National Health Service (NHS), ethical and medicolegal issues, receiving feedback from colleagues and the different learning strategies in the UK. IMGs suggested introduction of a mandatory dedicated induction program in the form of formal teaching sessions. They also believed that a supervised shadowing period prior in the first job in the UK would be beneficial. Further assessment areas should be incorporated into the prequalifying examinations to address specific educational needs such as NHS structure and hospital policies. Other measures such as buddying schemes with senior IMGs and educating NHS staff on different needs of IMGs should also be considered.

Conclusion: This study highlighted important educational challenges faced by IMGs and generated relevant solutions. However, the opinions of the supervisors and other health care professionals need to be explored.

Keywords: international medical graduates, IMG, educational barriers

\section{Introduction}

International medical graduates (IMGs) remain a unique and vital group of doctors in the UK. IMGs currently constitute approximately one-quarter of the General Medical Council (GMC) register. According to the 2012 statistics, $26 \%$ of all doctors registered in the GMC obtained their primary medical qualification (PMQ) overseas. ${ }^{1}$

IMGs come to the UK with the aim of advancing their careers and improving their clinical experience. ${ }^{2}$ They also seek better education, training and more exposure to the new developments and advances in the ever-evolving field of medicine.

Over the years, IMGs have been exposed to numerous obstacles to living and working in the UK. Many of these obstacles are not education related, such as immigration and financial and psychosocial issues. ${ }^{3}$ The educational literature with respect to educational and learning challenges faced by IMGs in the UK is scarce, as the vast 
majority of papers addressing this issue come from the USA, Canada and Australia.

This qualitative study aimed to explore the educational challenges faced by IMGs working in the medical specialties in the UK.

\section{Methods}

This study was conducted in the Kent, Surrey and Sussex (KSS) region in 2015, and all IMGs in core and higher medical training (senior house officers and registrars) were included. In this study, only IMGs from areas of non-UK, non-European Economic Area (EEA) were included in the sample. A survey was sent electronically to all IMGs who fulfilled the inclusion criteria. In addition, trainees were approached during their mandatory teaching days. The survey was pretested initially with three respondents to remove any ambiguity. Following the survey distribution, three one-to-one interviews were held. Participants for the interview phase were invited by email. The total number of IMGs fulfilling the criteria was 61 out of 422 trainees in medical training in the KSS region. Thematic analysis was applied to qualitative data, and qualitative responses from both the survey and interviews were merged together in the final analysis and presentation of themes. Ethical exemption was granted by the ethics committee of University College London (UCL) for this study. Written consent was obtained from those who participated in the interview phase. Interviews were recorded electronically, and the analysis of the transcript was undertaken by the author.

\section{Results}

Of the 61 eligible IMGs, 17 returned the questionnaire (28\%). Three participated in the interview phase. The majority (11 of 17) of the survey respondents obtained their PMQ in Asia (India or Pakistan). The IMGs were working across seven different specialties, and their grades varied from the first year to the sixth year of medical training (specialist trainees year 1 to year 6 ). The average time spent by IMGs working outside the UK was 2.3 years, but their experience ranged from 0 to 10 years. In comparison, IMGs spent at least 3 years in medical practice by the time of the study with an average UK work experience of 7.6 years.

Of the 17 survey respondents, eight (41\%) did not receive any formal induction when they initially worked in the UK. Of the remaining nine who received formal induction, only two indicated that the induction was relevant to their educational needs. The most common forms of induction were shadowing or attachment programs or induction days organized locally by hospitals.
The main barriers cited by the survey participants were lack of understanding of the hospital systems and the National Health Service (NHS), difficulties with receiving feedback with a poor relationship with supervisors, the different learning environment in the UK and dealing with ethical and medicolegal issues (Table 1).

When first started I was lost, I didn't know the hospital, I didn't know the system, I didn't know how things work and it wasn't easy at all. [Interview participant 1]

$[\ldots]$ just took me some time to come at ease with the cultural differences and how feedback is given. [Survey participant]

Back home there is no concept of "do not resuscitate" as such, but here it is, which is something new to me. [Interview participant 1]

Interestingly, difficulties with knowledge gaps and communications skills were not frequently mentioned. However, the interview participants highlighted that communication skills in difficult situations and inability to deal with medical emergencies also represented areas of insufficient confidence.

[It] put me into a difficult situation where the communication has affected me a lot. [Interview participant 2]

No sometimes the knowledge, yeah, your knowledge in dealing with emergencies in a practical way. [Interview participant 1]

Table I The major barriers faced by IMGs in this study

Lack of understanding of the NHS system and values

- Lack of experience in utilizing administrative protocols, hospital policies and guidelines

- Lack of understanding of the medicolegal framework in the UK

- Difficulty with integration into the new community of practice and work environment

The different learning environment in the UK

- Experiential learning in the UK vs. more didactic teaching in the country of origin

- Lack of direct guidance

- Need for more self-directed learning in the UK

Difficulty with receiving feedback

- Feedback perceived to be negative and associated with shame

- Poor relationship with supervisors

The feel of being undervalued by colleagues

- IMGs inappropriately perceived as incompetent doctors due to their lack of knowledge of the health care system

- IMGs feeling of occasionally treated as outsiders in the work environment

Others

- Communication skills in challenging situations (end-of-life and resuscitation decisions)

- Dealing with medical emergencies

- Handling ethical scenarios

Abbreviations: IMG, international medical graduate; NHS, National Health Service. 
The majority of IMGs did not feel that language skills were the cause for encountering difficulties in the UK. Nonetheless, they agreed that a lack of specific IMG induction programs, sociocultural differences and the different learning methods were among the major factors that led to challenges when beginning their first jobs in the UK health care system.

In addition to these factors, some IMGs attributed the barriers they encountered to a feeling of being undervalued by their colleagues, resulting occasionally in low self-esteem and lack of confidence (Table 1).

IMGs treated as second class doctors regardless of ability.

[Survey participant]

[...] you know, some considered me as, you know,

low class [doctor] or they preferred not to work with me.

[Interview participant 1]

The two principal suggestions made by IMGs were the introduction of mandatory supervised shadowing period with hands-on experience and a formal prolonged induction course with specific reference to the educational needs of IMGs (Table 2).

I think shadowing in a way that you are made to take some responsibility, not just observe. [Interview participant 1]

Possibly with a robust induction and time spent in clinical attachment. [Survey participant]

I think both, shadowing at the same time having proper, formal teaching sessions. [Interview participant 1]

Moreover, IMGs suggested restructuring the Professional and Linguistic Assessments Board (PLAB) exam to include more assessment areas on the structure of the NHS and health

Table 2 Suggestions for further action from the IMGs' perspective

Shadowing or clinical attachment

- Lasting for months

- Supervised hands-on experience

Induction program

- Lasting for days (or I week)

- Topics focusing on NHS values, medicolegal issues, smooth integration and transition, etc.

- Comparative teaching model

Improving supervision and mentorship

- Enhanced mentorship in the first job in the UK

- More frequent educational meetings to address on-going challenges

- Educating supervisors and consultants on IMG needs

Improvements to the PLAB exam

- Including more assessment areas on NHS values

Others

- Raising the awareness of NHS staff on IMG learning needs

- "Buddying system" between new and senior IMGs

- Counseling of IMGs prior to coming to the UK

Abbreviations: IMG, international medical graduate; NHS, National Health Service; PLAB, Professional and Linguistic Assessments Board. care policies in the UK. An enhanced mentorship program during their first job was also recommended by IMGs, which should be coupled with raising the awareness of supervisors and health care professionals on the special educational and learning needs of IMGs.

[PLABs] they prepare you reasonably well but PLAB 2 was also for the clinical side [like] clinics admission and so on, but there was no introduction to any NHS system, policies, guidelines, nothing mentioned of this. [Interview participant 1]

Educating NHS workers (Dr, Nurses, HCA etc) about challenges faced by IMG. [Survey participant]

Finally, some IMGs mentioned that they benefited from advice and guidance from the senior IMGs they met locally in overcoming the hurdles they faced in the health care system. This reflects the need for a formal "buddying system" whereby more settled IMGs can be approached to act as mentors for newly arrived IMGs.

Everybody is coming from different countries and working together. I think the support from the [IMG] colleagues; I felt that yeah, it has been a good support throughout .... [Interview participant 3]

\section{Discussion}

The cohort of IMGs in this study had a relatively long experience in the UK. The majority spent more time in UK medical practice than in their original countries with some having minimal or no clinical experience prior to joining the NHS. This finding may suggest that there is currently a general preference for some IMGs to establish their career in the UK at an earlier stage.

The major barrier for these IMGs appeared to be the lack of understanding of the health care system at all levels, including the various administrative protocols, hospital policies and community services. In their model of adaptation of IMGs to new health care systems, Wong and Lohfield ${ }^{4}$ described this phase as the loss and orientation phase of transition into new health care systems. In this phase, they believed that IMGs feel like outsiders in the medical training program and struggle to understand how to fit in as a result of the differences in the organization of the medical system in the new environment.

Unlike previous studies, in the current study, communication was not perceived to be the major barrier. The main difficulty in communication appeared to arise in sensitive and complex clinical scenarios. This anxiety was explored in depth by Slowther et al, ${ }^{5}$ who showed that non-UK qualified doctors experienced various challenges related to practicing within a different ethical regulatory framework. The best 
example illustrated by IMGs in the current study was that dealing with the medicolegal aspects of practicing medicine in the UK was one of the biggest fears for them. Their anxiety was more pronounced in situations that involved end-of-life and resuscitation decisions (Table 1).

The difficulty faced by IMGs when receiving feedback was an interesting finding. It could be explained, however, by the fact that many IMGs attain their PMQ in medical systems where feedback is often associated with shame and embarrassment. ${ }^{6}$

Most IMGs linked their educational barriers with sociocultural differences and misunderstandings. Social interactions in health care systems form an intrinsic part of clinical practice. $^{7}$ These interactions not only affect IMGs' relationships with their patients and colleagues but may also play an important role in widening the knowledge gap experienced by IMGs with regard to understanding the health care system in the UK. Owing to ethical constraints on this study, the psychosocial factors and their impact on the learning process of IMGs could not be covered in great depth. While extreme social isolation was not reported in this study, some of the participants experienced discrimination and the feeling of being undervalued or being inappropriately perceived as incompetent by colleagues. While this challenge was only cited by a few IMGs, it reflects that poor performance could be linked to low self-esteem as previously illustrated by Maslow $^{8}$ in his discussion of behaviorism learning theory.

The difference in learning strategies was highlighted by many IMGs. Most of the learning in clinical settings in the UK takes place through experiential learning and cognitive constructivism. Nevertheless, this model of learning may not be very familiar to IMGs who were mostly trained in a didactic learning environment based on a purely cognitive learning process.

Another major reason leading to the barriers faced by IMGs was the lack of a dedicated induction program. The quality of the induction program is to be primarily blamed. The trainees criticized the traditional approach of single-day induction courses and felt that these courses are not specific to their educational needs and may result in information overload. Hence, in order to improve the efficiency of these programs, their content should focus on the specific needs of IMGs with special reference to medicolegal and ethical issues as well as the values and ethos of the NHS. Moreover, the induction programs should last for more than 1 day, preferably a whole week.

Clinical shadowing or spending a period of clinical attachment is an important intervention that could be implemented to help IMGs settle easily into the NHS system. Shadowing as a method of induction of new doctors is not a new strategy and has been used previously in the NHS, particularly for newly qualified doctors joining the UK foundation program with successful outcomes. ${ }^{9}$ Similar studies suggested that longer periods of shadowing are more beneficial than the short 1-week shadowing. ${ }^{7,9}$

In addition, IMGs seem to believe that their transition into the NHS is a continuous process. While shadowing and induction offer assistance at the beginning of the transition process, enhancing their mentorship through more frequent meetings with their supervisors and raising NHS staff awareness of IMG needs are also required. This model of more focused supervision of IMGs in their first year has also been supported by other studies. ${ }^{5-7}$

One interesting suggestion made by IMGs is the need for a "buddying system" whereby IMGs are guided by senior IMG peers. This could represent a powerful form of peerassisted learning if implemented formally by deaneries and local trusts. In addition, senior IMGs can develop their skills as teachers and mentors for newly arrived IMGs.

\section{Conclusion}

This study not only underscored the various educational barriers faced by IMGs but also discovered and generated practical solutions. However, the views of supervisors on the recommendations made here by IMGs need to be explored prior to implementation. While sociocultural factors interact significantly with the educational barriers and other difficulties highlighted by IMGs, these could not be investigated in this study. Finally, the interviews were conducted by an IMG. Although this facilitated the discussion, analysis of the data by a non-IMG may have generated different themes.

\section{Disclosure}

The author reports no conflicts of interest in this work.

\section{References}

1. GMC. The State of Medical Education and Practice in the UK. London: General Medical Council; 2013.

2. Trewby PN. 'International medical graduates: lessons from the past hopes for the future'. Clin Med. 2008;8(3):283-287.

3. Chen PG, Curry LA, Bernheim SM, Berg D, Gozu A, Nunez-Smith M. Professional challenges of non-U.S.-born international medical graduates and recommendations for support during residency training. Acad Med. 2011;86(11):1383-1388.

4. Wong A, Lohfeld L. Recertifying as a doctor in Canada: international medical graduates and the journey from entry to adaptation. Med Educ. 2008;42(1):53-60.

5. Slowther A, Hundt LGA, Purkis J, Taylor R. 'Experiences of non-UK qualified doctors working within the UK regulatory framework: a qualitative study'. J R Soc Med. 2012;105(4):157-165. 
6. Pilotto LS, Duncan G, Anderson-Wurf J. 'Issues for clinicians training international medical graduates: a systematic review'. Med J Aust. 2007;187(4):225-228.

7. Rothwell C, Morrow G, Burford B, Illing J. Ways in which healthcare organisations can support overseas-qualified doctors in the UK. Int $J$ Med Educ. 2013;4:75-82.
8. Maslow A. Motivation and Personality. New York: Harper; 1954.

9. Rich AJ. 'An induction programme for first-appointment overseas doctors'. Med Teach. 1998;20:473-475.

\section{Publish your work in this journal}

Advances in Medical Education and Practice is an international, peerreviewed, open access journal that aims to present and publish research on Medical Education covering medical, dental, nursing and allied health care professional education. The journal covers undergraduate education, postgraduate training and continuing medical education including emerging trends and innovative models linking education, research, and health care services. The manuscript management system is completely online and includes a very quick and fair peer-review system. Visit http://www.dovepress.com/testimonials.php to read real quotes from published authors.

Submit your manuscript here: http://www.dovepress.com/advances-in-medical-education-and-practice-journal 